\title{
Description of the Charge Transfer States at the Pentacene/ C60 Interface: Combining Range-Separated Hybrid Functionals with the Polarizable Continuum Model
}

\author{
Zilong Zheng, Jean-Luc Bredas, and Veaceslav Coropceanu
}

J. Phys. Chem. Lett., Just Accepted Manuscript • DOI: 10.1021/acs.jpclett.6b00911 • Publication Date (Web): 24 Jun 2016

Downloaded from http://pubs.acs.org on June 28, 2016

\section{Just Accepted}

"Just Accepted" manuscripts have been peer-reviewed and accepted for publication. They are posted online prior to technical editing, formatting for publication and author proofing. The American Chemical Society provides "Just Accepted" as a free service to the research community to expedite the dissemination of scientific material as soon as possible after acceptance. "Just Accepted" manuscripts appear in full in PDF format accompanied by an HTML abstract. "Just Accepted" manuscripts have been fully peer reviewed, but should not be considered the official version of record. They are accessible to all readers and citable by the Digital Object Identifier (DOI®). "Just Accepted" is an optional service offered to authors. Therefore, the "Just Accepted" Web site may not include all articles that will be published in the journal. After a manuscript is technically edited and formatted, it will be removed from the "Just Accepted" Web site and published as an ASAP article. Note that technical editing may introduce minor changes to the manuscript text and/or graphics which could affect content, and all legal disclaimers and ethical guidelines that apply to the journal pertain. ACS cannot be held responsible for errors or consequences arising from the use of information contained in these "Just Accepted" manuscripts.

\section{ACS Publications}


Description of the Charge Transfer States at the Pentacene/ $C_{60}$

\title{
Interface: Combining Range-Separated Hybrid Functionals with the
}

\section{Polarizable Continuum Model}

\author{
Zilong Zheng, ${ }^{1}$ Jean-Luc Brédas, ${ }^{2 *}$ and Veaceslav Coropceanu ${ }^{1 *}$ \\ ${ }^{1}$ School of Chemistry and Biochemistry and Center for Organic Photonics and \\ Electronics Georgia Institute of Technology, Atlanta, Georgia 30332-0400 \\ ${ }^{2}$ Solar \& Photovoltaics Engineering Research Center, \\ Division of Physical Science and Engineering, \\ King Abdullah University of Science and Technology, \\ Thuwal 23955-6900, Saudi Arabia
}

*E-mails: jean-luc.bredas@kaust.edu.sa; coropceanu@gatech.edu 


\begin{abstract}
Density functional theory (DFT) approaches based on range-separated hybrid functionals are currently methods of choice for the description of the charge-transfer (CT) states in organic donor/acceptor solar cells. However, these calculations are usually performed on small-size donor/acceptor complexes and as result do not account for electronic polarization effects. Here, using a pentacene $/ \mathrm{C}_{60}$ complex as a model system, we discuss the ability of long-range corrected (LCR) hybrid functionals in combination with the polarizable continuum model (PCM) to determine the impact of the solid-state environment on the CT states. The CT energies are found to be insensitive to the interactions with the dielectric medium when a conventional time-dependent DFT/PCM (TDDFT/PCM) approach is used. However, a decrease in the energy of the CT state in the framework of LRC functionals can be obtained by using a smaller range-separated parameter when going from an isolated donor/acceptor complex to the solid-state case.
\end{abstract}




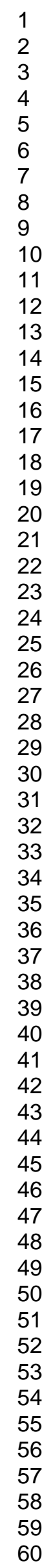

TOC Graphic

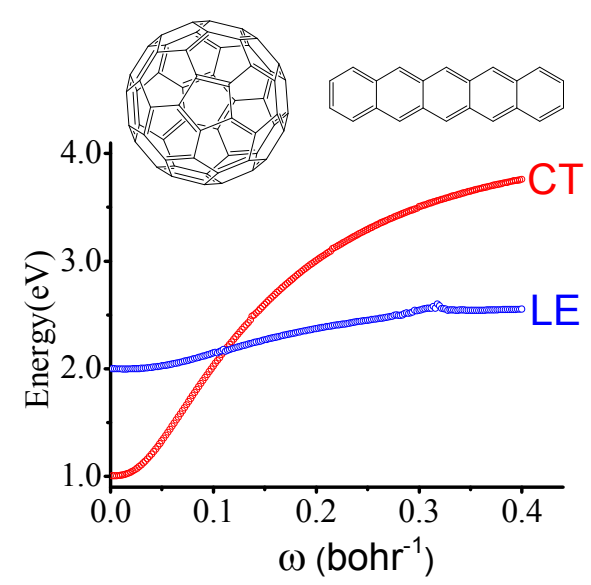

20

21

23

24

26

27

28
29

30

31

33

34

35

36

37

38

39

40

41

42

43
44

45

46

47

48

49

51

52

53

54

55

57

58

59

60 
In organic solar cells charge-transfer (CT) states at the interface between electron donor (D) and electron acceptor (A) materials play a major role in both exciton-dissociation and charge-recombination processes. ${ }^{1-4}$ Both processes are key for the performance of the photovoltaic cells and have therefore received significant attention from both experimental and theoretical sides.

While Density functional theory (DFT) is a method of choice for many quantum-mechanical applications, obtaining an accurate theoretical description of the interfacial CT states by means of DFT methods, remains challenging. Standard semi-local and global hybrid exchange-correlation functionals do not provide the correct evolution of the long-range Coulomb interactions and result in a spurious self-repulsion of charge densities. ${ }^{5-8}$ A proper description of the asymptotic, $1 / \mathrm{r}$, dependence can be obtained with range-separated hybrid (RSH) functionals. ${ }^{9}$ In the case of long-range corrected (LRC) functionals, a simple separation of the electron-electron interactions is based upon:

$$
\frac{1}{r}=\frac{\operatorname{erf}(\omega r)}{r}+\frac{\operatorname{erfc}(\omega r)}{r}
$$

where $\omega$ corresponds to the range-separation (RS) parameter. Here, Hartree-Fock (HF) exchange is employed to treat the long-range exchange (first term to the right of Eq. 1) while a local or semi-local DFT functional is used to treat the short-range exchange (second term to the right of Eq. 1).

It was shown that RSH functionals provide the best results for a given system when the RS parameter is optimally tuned. Several physically motivated tuning conditions are used in the literature. For instance, a widely used non-empirical tuning 
procedure is based upon minimizing the difference between the energy of the highest occupied molecular orbital (HOMO) and the first ionization potential (IP). ${ }^{10,11}$ The tuning procedures usually employ the results derived for an isolated system (in vacuo); we will refer to this approach below as DFT/ $\omega(\mathrm{vac})$.

Our recent studies on pentacene $/ \mathrm{C}_{60}$ complexes ${ }^{12,13}$ show that the $\mathrm{CT}$ energies calculated with default- $\omega$ LRC functionals could be as large as $3 \mathrm{eV}$ (i.e., about twice the experimental values ${ }^{14}$ ) and strongly depend on the choice of the functional. Upon tuning the range-separation parameter, all LRC functionals give comparable results for both local and CT excited states. The tuning also results in a significant (up to $1 \mathrm{eV}$ ) lowering of the CT energies. However, the DFT/w(vac) - based energy of the lowest CT state is still too high, with the CT state lying above the local excited states on pentacene and $\mathrm{C}_{60},{ }^{12,13}$ in contrast to experimental observations. ${ }^{14,15}$ Similar results have also been obtained by Nakano and co-workers. ${ }^{16,17}$ Our initial studies were based on complexes involving just one pentacene molecule and one $\mathrm{C}_{60}$ molecule; thus, the impact of wavefunction delocalization and electronic polarization effects could not be accounted for. We have found that increasing the size of the pentacene $/ \mathrm{C}_{60}$ complexes $^{12}$ (by including up to five pentacene molecules) results in some lowering of CT state energy. Thus, a further increase in the size of D/A clusters is expected to lead to an even better description of the role of electron delocalization and polarization effects on the nature of the CT states. Such calculations, however, quickly become very time consuming. In addition, tuning the range-separation parameter with increasing aggregate dimensions may result in size consistency errors. ${ }^{18,19}$ A computationally inexpensive way of considering the medium (electron polarization) effect on the CT states is to combine the DFT calculations with a continuum solvation approach such as the polarizable continuum model $(\mathrm{PCM}){ }^{20}$ 
There are two approaches to choose the RS parameters when RSH/PCM calculations are performed. The first approach is to use RS parameters optimally-tuned in vacuum and then to perform DFT calculations in the presence of dielectric medium; we refer to this approach as DFT/ $(\mathrm{vac}) / \mathrm{PCM}$. Alternatively, the RS parameter can be optimized, as discussed by de Queiroz and Kümmel, ${ }^{21}$ in the presence of the dielectric medium and the TDDFT/PCM calculations are then carried out with the PCM-tuned RS parameter; we refer to this approach as DFT/ $(\mathrm{PCM}) / \mathrm{PCM}$. However, the soundness of such a DFT/ $\omega(\mathrm{PCM}) / \mathrm{PCM}$ approach has been questioned. ${ }^{21,22}$ For instance, it was found that this approach underestimates the energies of intra-molecular CT states. More recently, de Queiroz and Kümmel ${ }^{23}$ proposed an approach that explicitly includes the solvent molecules into the tuning procedure. While this approach is more accurate than the $\mathrm{DFT} / \omega(\mathrm{PCM})$ approach, it is also more computationally demanding. We also note that in the context of D/A systems, the use of the explicit-solvent model would mean considering larger D/A complexes. In order to shed more light on this issue, we study here the performance of the DFT/ $\omega(\mathrm{vac}), \mathrm{DFT} / \omega(\mathrm{vac}) / \mathrm{PCM}$, and DFT/ $/ \mathrm{PCM}) / \mathrm{PCM}$ approaches on the description of the inter-molecular $\mathrm{CT}$ states using a pentacene $/ \mathrm{C}_{60}$ complex as model system.

The geometries of the isolated pentacene and $\mathrm{C}_{60}$ molecules were optimized at the B3LYP/6-31G(d,p) level. Following our previous studies, the optimized molecular geometries of pentacene and $\mathrm{C}_{60}$ were used to construct an "edge-on" pentacene $/ \mathrm{C}_{60}$ $\left(\mathrm{P} / \mathrm{C}_{60}\right)$ model complex with center-to-center intermolecular distance of $13 \AA$ (see Figure 1). The excited-state transition energies and oscillator strengths were obtained by means of both full time-dependent DFT (TDDFT) calculations and TDDFT calculations based on the Tamm-Dancoff approximation (TDA-TDDFT). ${ }^{24-26}$ In addition, the lowest $\mathrm{CT}$ energy of the $\mathrm{P} / \mathrm{C}_{60}$ complex was obtained by means of a 
constrained DFT (CDFT) approach. ${ }^{27,28}$ The impact of HF exchange was also investigated by using the $\alpha \mathrm{PBE}$ functional, based on the original PBE functional, ${ }^{29,30}$ following the simple hybrid scheme proposed by Adamo and Barone: ${ }^{31}$

$$
E_{\alpha P B E}=\alpha E_{X}^{H F}+(1-\alpha) E_{X}^{P B E}+E_{C}^{P B E}
$$

where $\alpha=0.25$ corresponds to the PBE0 functional.

The DFT calculations were performed for an isolated complex (in vacuo) and in the presence of a dielectric medium using the polarizable continuum model (DFT/PCM). A dielectric constant $\varepsilon=3$ was used in all DFT/PCM calculations. The excited-state calculations were performed using the $\omega \mathrm{B} 97 \mathrm{X}-\mathrm{D}^{32}$ and $\mathrm{BNL}{ }^{19,33}$ LRC functionals with the 6-31G(d,p) basis set. All DFT calculations were performed with the Q-Chem 4 package. $^{34,35}$

The $\omega$ value was optimized by minimizing the expression $\mathrm{J}(\omega):^{10}$

$$
\mathrm{J}=\left(\mathrm{E}_{\mathrm{HOMO}}-\mathrm{IP}\right)^{2}+\left(\mathrm{E}_{\mathrm{LUMO}}-\mathrm{EA}\right)^{2}
$$

Here, $\mathrm{E}_{\mathrm{HOMO}}$ and $\mathrm{E}_{\mathrm{LUMO}}$ denote the energies of the HOMO and LUMO (highest occupied and lowest unoccupied molecular orbitals); IP and EA are the vertical first ionization potential and electron affinity of the system, respectively.

Since the calculations using the $\omega \mathrm{B} 97 \mathrm{XD}$ and BNL functionals yield very similar results, only the $\omega \mathrm{B} 97 \mathrm{XD}$ results are discussed hereafter (the results derived on the basis of the BNL functional can be found in the Supporting Information (SI, Table S1). The tuned values of $\omega$ for the $\omega$ B97XD functional are listed in Table 1. In agreement with earlier work, ${ }^{21}$ the values of RS parameter calculated at the DFT/ $\omega(\mathrm{PCM}) / \mathrm{PCM}$ level, are much smaller (about 7 times) than those tuned for the isolated systems

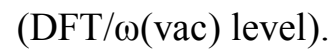


Table 1. Optimized $\omega$ values [in bohr ${ }^{-1}$ ] of pentacene, $\mathrm{C}_{60}$, and the $\mathrm{P} / \mathrm{C}_{60}$ complex, at the $\omega \mathrm{B} 97 \mathrm{XD} / 6-31 \mathrm{G}(\mathrm{d}, \mathrm{p})$ level.

\begin{tabular}{cc|c}
\hline \hline & $\omega($ vacuum $)$ & $\omega(\mathrm{PCM})$ \\
\hline Pentacene & 0.165 & 0.025 \\
$\mathrm{C}_{60}$ & 0.178 & 0.023 \\
Pentacene $/ \mathrm{C}_{60}$ & 0.148 & 0.021 \\
\hline \hline
\end{tabular}

The computed HOMO and LUMO energies of both molecules and of the $\mathrm{P} / \mathrm{C}_{60}$ complex are shown in Figure 1. As seen from this figure, the account of the interaction with the dielectric environment has only a marginal affect (see also Table S2 in SI) on the frontier orbital energies when the $\omega(\mathrm{vac})$ parameters are used. However, the calculations at the DFT/ $\omega(\mathrm{PCM}) / \mathrm{PCM}$ level lead to a significant change $(1 \mathrm{eV}$ or larger) in the orbital energies, which is consistent with the experimental data. ${ }^{36,37}$
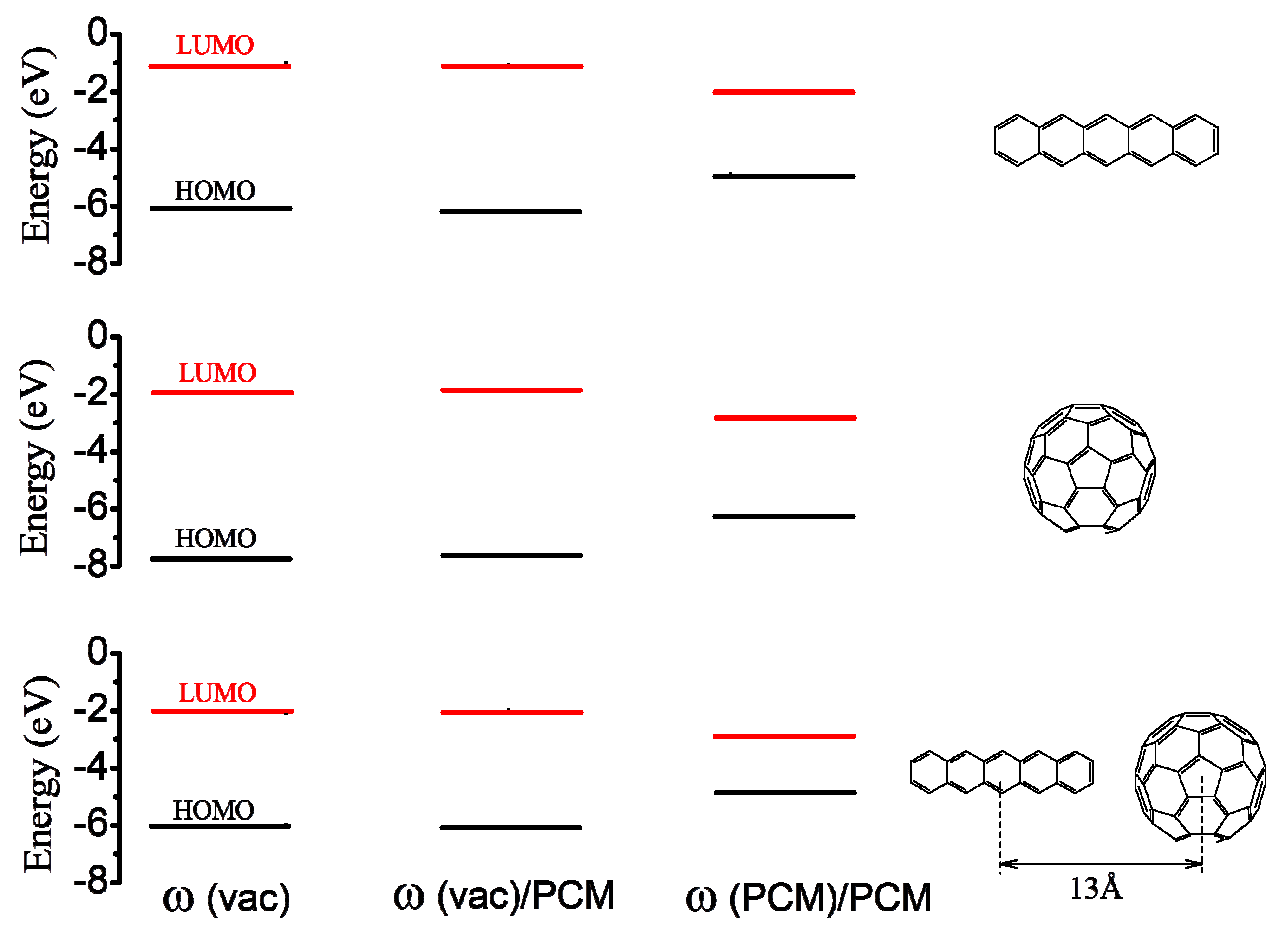

Figure 1. Energies of the HOMO and LUMO levels of pentacene, $\mathrm{C}_{60}$ and the pentacene $/ \mathrm{C}_{60}$ complex at the $\omega \mathrm{B} 97 \mathrm{XD} / 6-31 \mathrm{G}(\mathrm{d}, \mathrm{p})$ level. 
The energies of the lowest excited state of pentacene, $\mathrm{C}_{60}$, and the $\mathrm{P} / \mathrm{C}_{60}$ complex derived at both TDDFT and TDA-TDDFT levels, are shown in Table 2. The experimental value for the pentacene lowest excited singlet state $\left(\mathrm{S}_{1}\right)$ energy is about $2.10 \mathrm{eV}$ in solution, ${ }^{38} 1.85 \mathrm{eV}$ in the solid state, ${ }^{39,40}$ and $2.31 \mathrm{eV}$ in the gas phase. ${ }^{41}$ The fluorescence spectra for an isolated $C_{60}$ molecule suggest that the origin of the $S_{0} \rightarrow S_{1}$ transition is at $c a .1 .94 \mathrm{eV} ;^{42}$ in the solid state, this value reduces to $1.7 \mathrm{eV} .^{43}$ The TDDFT energies are in good agreement with experiment in the case of pentacene but somewhat higher than the experimental values in the case of $\mathrm{C}_{60}$. As in the case of the molecular orbital energies, there are only very small differences between the

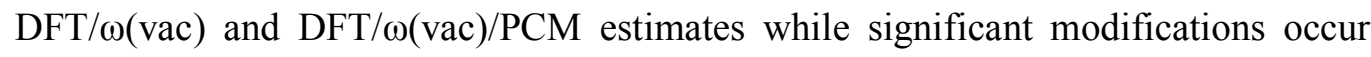
when going to the DFT/ $(\mathrm{PCM}) / \mathrm{PCM}$ level. The most critical change is seen in the

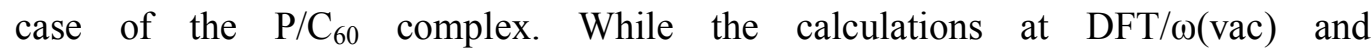
$\mathrm{DFT} / \omega(\mathrm{vac}) / \mathrm{PCM}$ levels predict that the first excited state (at $2.2-2.3 \mathrm{eV}$ ) is a local pentacene-based state, in contrast, the first excited state at the DFT/ $(\mathrm{PCM}) / \mathrm{PCM}$ level has a CT character. Overall the calculations at the full TDDFT level and at the TDA-TDDFT level provide very similar the descriptions of the excited states. The lowest CT state according to both TDDFT and TDA-TDDFT calculations is located at ca. $1.2 \mathrm{eV}$, which matches the range of experimental estimates for CT energies in D/A heterojunctions. ${ }^{14}$ Our calculations thus indicate that in the case of $\mathrm{P} / \mathrm{C}_{60}$, the $\mathrm{DFT} / \omega(\mathrm{PCM}) / \mathrm{PCM}$ approach correctly describes the qualitative effect of the dielectric medium on both frontier orbital energies and CT energies. This result is of 
course related to the fact that the RS parameters used for the isolated system and for the system imbedded in a dielectric medium are substantially different.

Table 2. TDDFT and TDA-TDDFT energies (in $\mathrm{eV}$ ) and oscillator strengths (given in parentheses) of the first excited state of pentacene, $\mathrm{C}_{60}$, and the $\mathrm{P} / \mathrm{C}_{60}$ complex.

\begin{tabular}{ccc|c|c}
\hline \hline & & $\omega(\mathrm{vac})$ & $\omega(\mathrm{vac}) / \mathrm{PCM}$ & $\omega(\mathrm{PCM}) / \mathrm{PCM}$ \\
\hline \multirow{4}{*}{ TDDFT } & Pentacene & $2.30(0.07)$ & $2.24(0.10)$ & $1.95(0.06)$ \\
& $\mathrm{C}_{60}$ & $2.56(0.00)$ & $2.56(0.00)$ & $2.16(0.00)$ \\
& $\mathrm{P} / \mathrm{C}_{60}$ & $2.26(0.06)$ & $2.21(0.09)$ & $1.24(0.00)$ \\
\hline \multirow{4}{*}{ TDA-TDDFT } & Pentacene & $2.62(0.14)$ & $2.52(0.18)$ & $2.19(0.12)$ \\
& $\mathrm{C}_{60}$ & $2.61(0.00)$ & $2.61(0.00)$ & $2.16(0.00)$ \\
& $\mathrm{P} / \mathrm{C}_{60}$ & $2.52(0.00)$ & $2.48(0.17)$ & $1.24(0.00)$ \\
\hline \hline
\end{tabular}

In order to get a better understanding of the role that the interplay between the medium effect and the choice of the RS parameter plays on the system energetics, we illustrate in Figure 2 the evolutions of the frontier orbital energies and IP and EA values (computed at the $\triangle \mathrm{SCF}$ level) in pentacene (Figure 2a), $\mathrm{C}_{60}$ (Figure $2 \mathrm{~b}$ ), and the $\mathrm{P} / \mathrm{C}_{60}$ complex (Figure $2 \mathrm{c}$ ), as a function of the $\mathrm{RS}$ parameter. Figure 2 , in agreement with previous findings, ${ }^{21,22}$ shows that the interaction with the dielectric medium (at least in the PCM approach used here) has only a minor effect on the orbital energies for all values of $\omega$ (the red and black lines corresponding to the HOMO and LUMO values are basically superimposed in Figure 2); however, the dielectric medium has a significant effect on, and markedly shifts the IP and EA values. In contrast, the orbital energies depend very strongly on the value of the RS parameter while the IP and EA show only a moderate dependence on $\omega$. It is clear that these results originate from the change in the amount of HF exchange when $\omega$ is 
modified. Indeed, as seen from Figure 3, the orbital energies computed, for example, with the $\alpha$ PBE functional scale linearly with the amount of HF exchange while the IP and EA again show only a modest variation. Thus, the increase in the amount of HF exchange from 0 to $100 \%$ leads to an increase in the HOMO-LUMO gap from $1.2 \mathrm{eV}$ to $6.42 \mathrm{eV}$, while only a minor increase of $0.05 \mathrm{eV}$ is found for the fundamental gap (obtained as EA-IP). The orbital energies are not affected by the inclusion of the dielectric medium into the calculations and intersect the EA(PCM) and IP(PCM) lines in Figure 3 around $\alpha=0.33$. An interesting consequence is that it is then not surprising that the HOMO and LUMO energies derived by means of a hybrid functional such as B3LYP $(\%$ HF exchange= $=20 \%)$ and PBE0 $(\%$ HF exchange= $=25 \%)$ better compare with the IP and EA values derived in the solid state rather than with measurements on isolated molecules. This finding can be explained by the fact that, in the framework of the $\alpha \mathrm{PBE}$ functional (and other hybrid functionals), the long-range Coulomb interaction behaves as $\alpha / \mathrm{r}$ and thus accounts in a way for the dielectric screening. ${ }^{44}$ Interestingly, the derived value of $1 / \alpha \approx 3$ (see Figure 3 ) is consistent with the value of the dielectric constant $(\varepsilon=3)$ used in the PCM calculations. Similar conclusions have been reached by Sun et al. in the case of organic molecular crystals. ${ }^{45}$ 

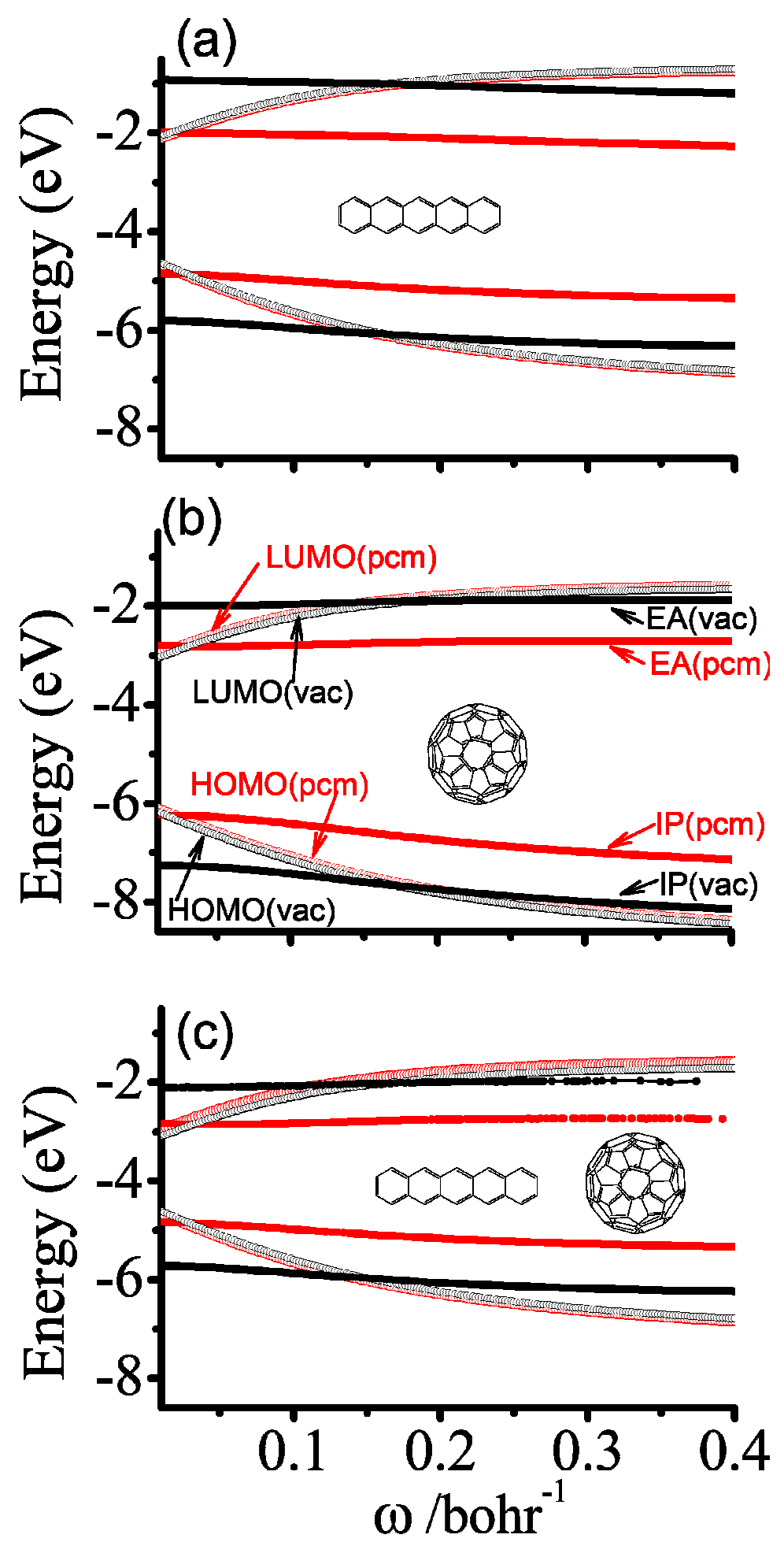

Figure 2. Dependence of the IP, EA, HOMO, and LUMO energies of pentacene (a), $\mathrm{C}_{60}$ (b), and the pentacene $/ \mathrm{C}_{60}$ complex (c), as a function of the $\mathrm{RS}$ parameter derived in vacuum (black lines) and the dielectric medium (red lines). 


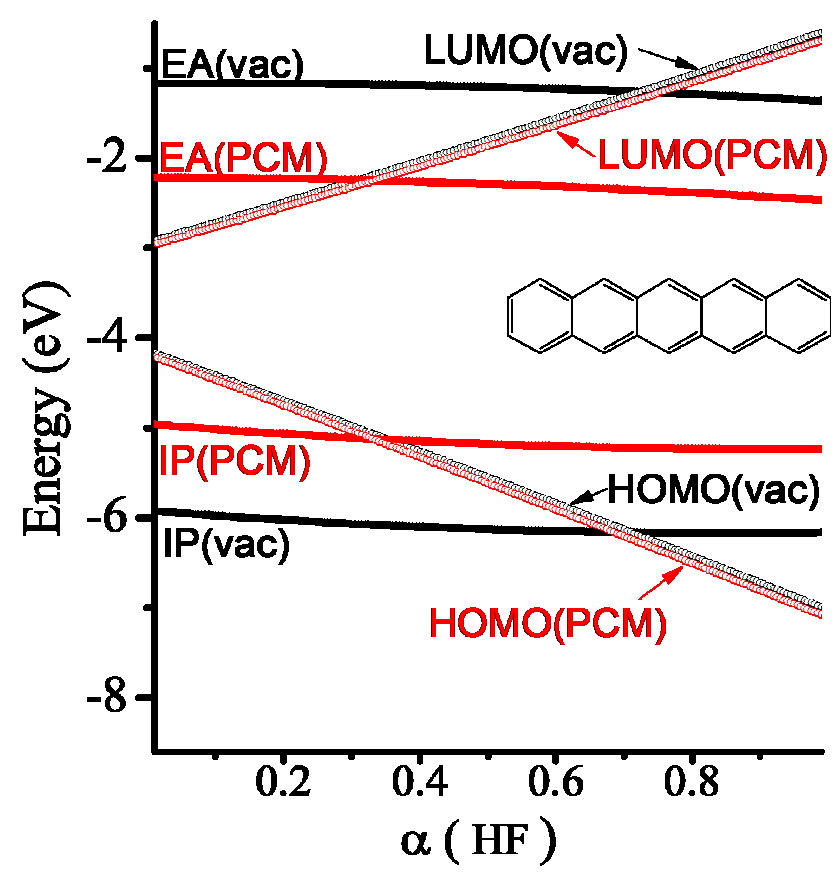

Figure 3. Dependence of the IP, EA, HOMO, and LUMO energies of the pentacene on the amount of HF exchange included in the $\alpha \mathrm{PBE}$ functional.

It is also useful to note that, in order to account for the solid-state environment, Phillips et $a l^{46}$ recently proposed an approach in which "environmentally-corrected" frontier orbital energies are obtained by adding to the DFT/ $\omega(\mathrm{vac})$ values a correction derived as the $[\mathrm{IP}(\mathrm{vac})-\mathrm{IP}(\mathrm{PCM})]$ or $[\mathrm{EA}(\mathrm{vac})-\mathrm{EA}(\mathrm{PCM})]$ difference. The "environmentally-corrected" energies derived in this way are, in fact, nearly identical to the $\mathrm{DFT} / \omega(\mathrm{PCM}) / \mathrm{PCM}$ values (as can be deduced from Figure 2). In the same context, Refaely-Abramson et $a l^{44}$ proposed an approach for the gap renormalization in molecular crystals by employing a density functional capable of capturing the effect of electronic polarization . 
We now turn to an analysis of how the choice of the RS parameter affects the excited-state energies of the $\mathrm{P} / \mathrm{C}_{60}$ complex. The evaluations of the lowest $\mathrm{CT}$-state energy and of the lowest local excited (LE) state energy as a function of $\omega$ are shown in Figure 4(a-b). The results obtained at the TDA-TDDFT (Figure 4a) and TDDFT (Figure $4 b$ ) levels are again very similar and indicate that, as in the case of the orbital energies, the energies of both LE and CT states are only slightly affected by the interaction with the dielectric medium. Interestingly, the $\mathrm{CT}(\mathrm{PCM})$ state is even located above the CT state derived for the isolated complex (in vacuo). As seen from Figure 4 , an increase in $\omega$ results in an increase in the energies of both CT and LE states. However, while the CT state energy shows a very strong evolution, only a moderate increase with $\omega$ is observed for the LE energy. As a consequence, if the CT state is located below the LE state at small $\omega$, the ordering of the CT and LE levels can change as $\omega$ increases. The reason why LE excitations and CT excitations show so markedly different evolutions with respect to $\omega$ is related to the fact that the CT states are very sensitive to the nature of the asymptotic potential, which is strongly affected by the choice of $\omega$, whereas the LE states probe the asymptotic potential to a much smaller degree. ${ }^{10,47}$ 

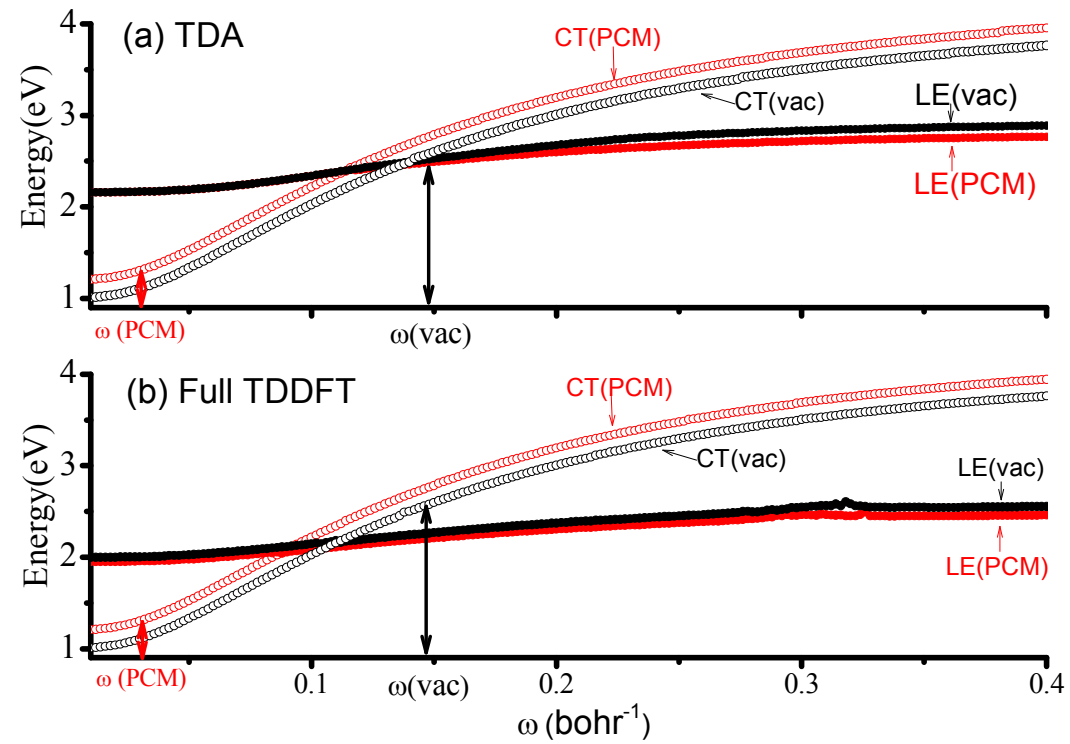

Figure 4. Dependence of the energy of the lowest CT state and the lowest local excited (LE) state of the $\mathrm{P} / \mathrm{C}_{60}$ complex, as a function of the RS parameter obtained via: (a) TDA-TDDFT and (b) TDDFT calculations, in vacuo (black lines) and in a dielectric medium (red lines).

In order to understand how the choice of the RS parameter impacts the CT excitations if they are derived at a $\triangle \mathrm{SCF}$ level, we show in Figure 5 the dependence of the CT-state energy as a function of $\omega$ obtained by means of a charge constrained DFT (CDFT) approach in vacuo and in the presence of the dielectric medium. The calculations indicate that, in contrast to TDDFT, the CDFT results are strongly affected by the consideration of the dielectric medium. The CDFT results yield a stabilization energy $\left[\mathrm{E}_{\mathrm{CT}}(\mathrm{vac})-\mathrm{E}_{\mathrm{CT}}(\mathrm{PCM})\right]$ of about $1.1 \mathrm{eV}$, which is essentially independent of $\omega$. This trend is similar to those found for the IP and EA values in Figure 2. When the $\mathrm{E}_{\mathrm{CT}}$ value of $2.57 \mathrm{eV}$ derived at the TDDFT/ $(\mathrm{vac})$ level is corrected by the $1.1 \mathrm{eV}$ stabilization energy derived above, we obtain a value of 1.47 
$\mathrm{eV}$ for the CT energy in the presence of dielectric medium, which is close to the value of $1.24 \mathrm{eV}$ derived at the TDDFT/ $/ \mathrm{PCM}) / \mathrm{PCM}$ level. The energies of the CT states shown in Figure 5 are largely overestimated. We note that they strongly depend on the amount of electron transferred from donor to acceptor and decrease as this amount decreases, see Figure S1 in the SI.

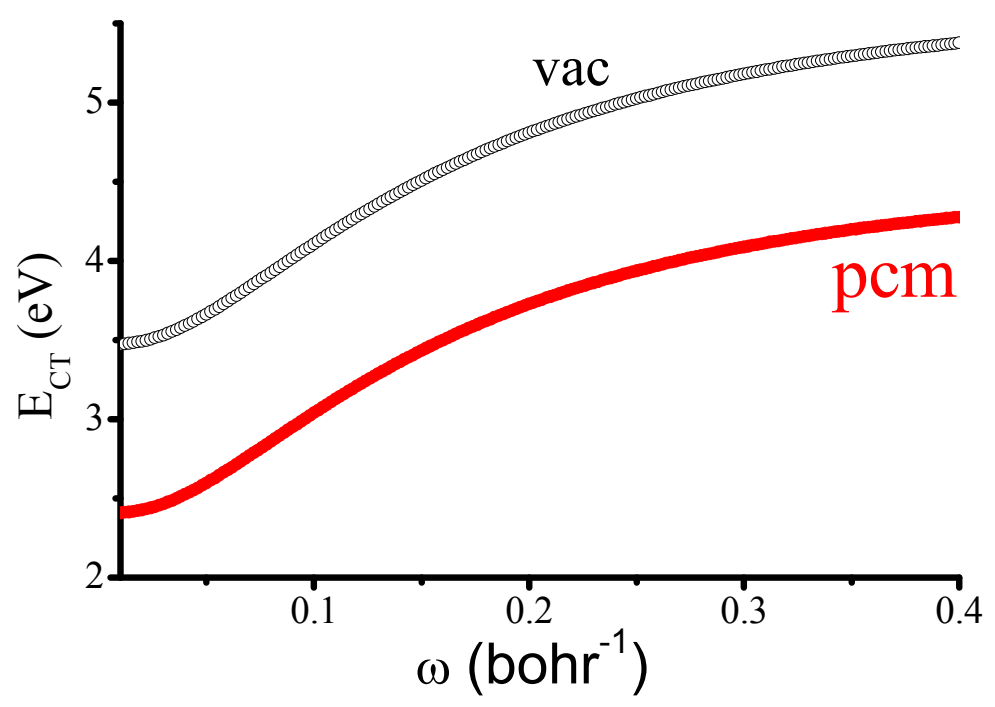

Figure 5. Dependence of the energy of the lowest $\mathrm{CT}$ state in the $\mathrm{P} / \mathrm{C}_{60}$ complex as a function of the RS parameter obtained by means of constrained DFT (CDFT) in vacuo (black line) and dielectric medium (red line), for 1 (Becke charge) electron transferred from donor to acceptor.

The comparison of the DFT and CDFT results indicates that when the IP, EA, and $\mathrm{CT}$ energies are derived at the $\triangle \mathrm{SCF}$ level, they show a correct stabilization due to the interaction with the dielectric environment. In the framework of the LRC functionals, these quantities can be derived by using the same RS parameter for both the isolated system and the system embedded in a dielectric medium. 
In this work, we investigated the performance of a combined density functional theory/polarizable continuum model (DFT/PCM) approach in describing the energetics of charge-transfer states in donor/acceptor complexes. In particular, we compared the results derived by means of long-range corrected functionals with range-separated parameters optimally tuned both in vacuo and in the presence of a dielectric medium.

Our results indicate that when the ionization potential, electron affinity and charge transfer (CT) state energies are derived by means of a $\triangle \mathrm{SCF}$ approach, the energy stabilization of these quantities due to the interaction with the dielectric environment is correctly described. However, we found that conventional time dependent (TD) DFT/PCM approaches fail in describing the impact of electron polarization on the CT states. We attribute this result to the fact that the orbital energies computed with and without inclusion of the interaction with the dielectric medium are nearly identical. However, these energies are strongly dependent on the amount of Hartree-Fock (HF) exchange included in the DFT functional. In a donor/acceptor system, a decrease in the amount of HF exchange results in a decrease in the HOMO-LUMO gap and, as a consequence, in the lowering of the $\mathrm{CT}$ energies. In the case of long-range corrected functionals, the amount of HF exchange can be controlled by adjusting the range-separated parameter. Therefore, in order to lower the HOMO-LUMO gap and the energy of the CT states, a smaller range-separated parameter needs to be considered when going from an isolated molecule or complex to the solid state. Finally, we note that, while our results point out that $\mathrm{CT}$ excitations in the $\mathrm{P} / \mathrm{C}_{60}$ complex can be 
reasonably well described by the $\mathrm{DFT} / \omega(\mathrm{PCM})$ approach, more systems should be investigated. It will be also important to test other models that are capable to account better for the effect of electronic polarization on both local and CT states.

Acknowledgements: The authors are most indebted to Dr. Leeor Kronik for very stimulating discussions. We gratefully acknowledge financial support of this work at the Georgia Institute of Technology by the Department of the Navy, Office of Naval Research (Award No. N00014-14-1-0580), and by King Abdullah University of Science and Technology (V.C.). The work at King Abdullah University of Science and Technology has been supported by KAUST competitive research funding and the Office of Naval Research - Global (Award No. N62909-15-1-2003).

Supporting Information Available: Energies of HOMO, LUMO, IP, and EA of pentacene, $\mathrm{C}_{60}$, and the pentacene/ $\mathrm{C}_{60}$ complex, using the optimally tuned $\mathrm{BNL} / 6-31 \mathrm{G}(\mathrm{d}, \mathrm{p})$ and $\omega \mathrm{B} 97 \mathrm{XD} / 6-31 \mathrm{G}(\mathrm{d}, \mathrm{p})$ functionals; dependence of the energy of the lowest $\mathrm{CT}$ state of the pentacene/ $\mathrm{C}_{60}$ complex as a function of the $\mathrm{RS}$ parameter obtained by means of constrained DFT for different amounts of (Becke) charge transferred from donor to acceptor. 
References

(1) Bredas, J. L., Norton, J. E., Cornil, J., Coropceanu, V. Molecular Understanding of Organic Solar Cells: The Challenges. Acc. Chem. Res. 2009, 42, 1691-1699.

(2) Vandewal, K., Albrecht, S., Hoke, E. T., Graham, K. R., Widmer, J., Douglas, J. D., Schubert, M., Mateker, W. R., Bloking, J. T., Burkhard, G. F. et al. Efficient Charge Generation by Relaxed Charge-Transfer States at Organic Interfaces. Nat. Mater. 2014, 13, 63-68.

(3) Gelinas, S., Rao, A., Kumar, A., Smith, S. L., Chin, A. W., Clark, J., van der Poll, T. S., Bazan, G. C., Friend, R. H. Ultrafast Long-Range Charge Separation in Organic Semiconductor Photovoltaic Diodes. Science 2014, 343, 512-516.

(4) Beljonne, D., Cornil, J., Muccioli, L., Zannoni, C., Bredas, J. L., Castet, F. Electronic Processes at Organic-Organic Interfaces: Insight from Modeling and Implications for Opto-Electronic Devices. Chem. Mater. 2011, 23, 591-609.

(5) Cohen, A. J., Mori-Sánchez, P., Yang, W. Challenges for Density Functional Theory. Chem. Rev. 2011, 112, 289-320.

(6) Becke, A. D. Density-Functional Thermochemistry. 3. The Role of Exact Exchange. J. Chem. Phys. 1993, 98, 5648-5652.

(7) Lee, C. T., Yang, W. T., Parr, R. G. Development of the Colle-Salvetti Correlation-Energy Formula into a Functional of the Electron-Density. Phys. Rev. B 1988, 37, 785-789.

(8) Stephens, P. J., Devlin, F. J., Chabalowski, C. F., Frisch, M. J. Ab Initio Calculation of Vibrational Absorption and Circular Dichroism Spectra Using Density Functional Force Fields. J. Phys. Chem. 1994, 98, 11623-11627.

(9) Leininger, T., Stoll, H., Werner, H. J., Savin, A. Combining Long-Range Configuration Interaction with Short-Range Density Functionals. Chem. Phys. Lett. 1997, 275, 151-160.

(10) Stein, T., Kronik, L., Baer, R. Reliable Prediction of Charge Transfer Excitations in Molecular Complexes Using Time-Dependent Density Functional Theory. J. Am. Chem. Soc. 2009, 131, 2818-2820.

(11) Stein, T., Kronik, L., Baer, R. Prediction of Charge-Transfer Excitations in Coumarin-Based Dyes Using a Range-Separated Functional Tuned from First Principles. J. Chem. Phys. 2009, 131, 244119.

(12) Yang, B., Yi, Y. P., Zhang, C. R., Aziz, S. G., Coropceanu, V., Bredas, J. L. Impact of Electron De Localization on the Nature of the Charge-Transfer States in Model Pentacene $/ \mathrm{C}_{60}$ Interfaces: A Density Functional Theory Study. J. Phys. Chem. C 2014, 118, 27648-27656.

(13) Zhang, C. R., Sears, J. S., Yang, B., Aziz, S. G., Coropceanu, V., Bredas, J. L. Theoretical Study of the Local and Charge-Transfer Excitations in Model Complexes of Pentacene- $\mathrm{C}_{60}$ Using Tuned Range-Separated Hybrid Functionals. J. Chem. Theory Comput. 2014, 10, 2379-2388. 
(14) Graham, K. R., Erwin, P., Nordlund, D., Vandewal, K., Li, R., Ngongang Ndjawa, G. O., Hoke, E. T., Salleo, A., Thompson, M. E., McGehee, M. D. et al. Re-Evaluating the Role of Sterics and Electronic Coupling in Determining the Open-Circuit Voltage of Organic Solar Cells. Adv. Mater. 2013, 25, 6076-6082.

(15) Beenken, W. J. D., Herrmann, F., Presselt, M., Hoppe, H., Shokhovets, S., Gobsch, G., Runge, E. Sub-Bandgap Absorption in Organic Solar Cells: Experiment and Theory. Phys. Chem. Chem. Phys. 2013, 15, 16494-16502.

(16) Minami, T., Nakano, M., Castet, F. Nonempirically Tuned Long-Range Corrected Density Functional Theory Study on Local and Charge-Transfer Excitation Energies in a Pentacene/ $\mathrm{C}_{60}$ Model Complex. J. Phys. Chem. Lett. 2011, 2, 1725-1730.

(17) Minami, T., Ito, S., Nakano, M. Functional Dependence of Excitation Energy for Pentacene $/ \mathrm{C}_{60}$ Model Complex in the Nonempirically Tuned Long-Range Corrected Density Functional Theory. Int. J. Quantum Chem. 2013, 113, 252-256.

(18) Karolewski, A., Kronik, L., Kummel, S. Using Optimally Tuned Range Separated Hybrid Functionals in Ground-State Calculations: Consequences and Caveats. J. Chem. Phys. 2013, 138, 204115.

(19) Livshits, E., Baer, R. A Well-Tempered Density Functional Theory of Electrons in Molecules. Phys. Chem. Chem. Phys. 2007, 9, 2932-2941.

(20) Mennucci, B., Tomasi, J. Continuum Solvation Models: A New Approach to the Problem of Solute's Charge Distribution and Cavity Boundaries. J. Chem. Phys. 1997, 106, 5151-5158.

(21) de Queiroz, T. B., Kummel, S. Charge-Transfer Excitations in Low-Gap Systems under the Influence of Solvation and Conformational Disorder: Exploring Range-Separation Tuning. J. Chem. Phys. 2014, 141, 084303

(22) Bokareva, O. S., Grell, G., Bokarev, S. I., Kuhn, O. Tuning Range-Separated Density Functional Theory for Photocatalytic Water Splitting Systems. J. Chem. Theory Comput. 2015, 11, 1700-1709.

(23) de Queiroz, T. B., Kummel, S. Tuned Range Separated Hybrid Functionals for Solvated Low Bandgap Oligomers. J. Chem. Phys. 2015, 143, 034101.

(24) Wang, Y. L., Wu, G. S. Improving the Tddft Calculation of Low-Lying Excited States for Polycyclic Aromatic Hydrocarbons Using the Tamm-Dancoff Approximation. Int. J. Quantum Chem. 2008, 108, 430-439.

(25) Hsu, C. P., Hirata, S., Head-Gordon, M. Excitation Energies from Time-Dependent Density Functional Theory for Linear Polyene Oligomers: Butadiene to Decapentaene. J. Phys. Chem. A 2001, 105, 451-458.

(26) Hirata, S., Head-Gordon, M. Time-Dependent Density Functional Theory within the Tamm-Dancoff Approximation. Chem. Phys. Lett. 1999, 314, 291-299.

(27) Wu, Q., Van Voorhis, T. Constrained Density Functional Theory and Its Application in Long-Range Electron Transfer. J. Chem. Theory Comput. 2006, 2, 765-774. 
(28) Wu, Q., Van Voorhis, T. Direct Calculation of Electron Transfer Parameters through Constrained Density Functional Theory. J. Phys. Chem. A 2006, 110, 9212-9218.

(29) Perdew, J. P., Burke, K., Ernzerhof, M. Generalized Gradient Approximation Made Simple. Phys. Rev. Lett. 1996, 77, 3865.

(30) Perdew, J. P., Burke, K., Ernzerhof, M. Erratum: Generalized Gradient Approximation Made Simple. Phys. Rev. Lett. 1997, 78, 1396.

(31) Adamo, C., Barone, V. Toward Reliable Density Functional Methods without Adjustable Parameters: The PBE0 Model. J. Chem. Phys. 1999, 110, 6158-6170.

(32) Chai, J. D., Head-Gordon, M. Long-Range Corrected Hybrid Density Functionals with Damped Atom-Atom Dispersion Corrections. Phys. Chem. Chem. Phys. 2008, 10, 6615-6620.

(33) Baer, R., Neuhauser, D. Density Functional Theory with Correct Long-Range Asymptotic Behavior. Phys. Rev. Lett. 2005, 94, 043002.

(34) Krylov, A. I., Gill, P. M. W. Q-Chem: An Engine for Innovation. Wiley Interdisciplinary Reviews: Computational Molecular Science 2013, 3, 317-326.

(35) Shao, Y., Molnar, L. F., Jung, Y., Kussmann, J., Ochsenfeld, C., Brown, S. T., Gilbert, A. T. B., Slipchenko, L. V., Levchenko, S. V., O'Neill, D. P. et al. Advances in Methods and Algorithms in a Modern Quantum Chemistry Program Package. Phys. Chem. Chem. Phys. 2006, 8, 3172-3191.

(36) Hwang, J., Wan, A., Kahn, A. Energetics of Metal-Organic Interfaces: New Experiments and Assessment of the Field. Mater. Sci. Eng. R 2009, 64, 1-31.

Griffith, O. L., Jones, A. G., Anthony, J. E., Lichtenberger, D. L. Intermolecular Effects on the Hole States of Triisopropylsilylethynyl-Substituted Oligoacenes. J. Phys. Chem. C 2010, 114, 13838-13845.

(38) Sakamoto, Y., Suzuki, T., Kobayashi, M., Gao, Y., Fukai, Y., Inoue, Y., Sato, F., Tokito, S. Perfluoropentacene: High-Performance P-N Junctions and Complementary Circuits with Pentacene. J. Am. Chem. Soc. 2004, 126, 8138-8140.

(39) Faltermeier, D., Gompf, B., Dressel, M., Tripathi, A. K., Pflaum, J. Optical Properties of Pentacene Thin Films and Single Crystals. Phys. Rev. B 2006, 74, 125416.

(40) Jundt, C., Klein, G., Sipp, B., Lemoigne, J., Joucla, M., Villaeys, A. A. Exciton Dynamics in Pentacene Thin-Films Studied by Pump-Probe Spectroscopy. Chem. Phys. Lett. 1995, 241, 84-88.

(41) Heinecke, E., Hartmann, D., Muller, R., Hese, A. Laser Spectroscopy of Free Pentacene Molecules (I): The Rotational Structure of the Vibrationless $\mathrm{S}_{1} \leftarrow \mathrm{S}_{0}$ Transition. J. Chem. Phys. 1998, 109, 906-911.

(42) Orlandi, G., Negri, F. Electronic States and Transitions in $\mathrm{C}_{60}$ and $\mathrm{C}_{70}$ Fullerenes. Photochem. Photobiol. Sci. 2002, 1, 289-308.

(43) Akimoto, I., Ashida, M., Kan'no, K. Luminescence from $\mathrm{C}_{60}$ Single Crystals in 
Glassy Phase under Site-Selective Excitation. Chem. Phys. Lett. 1998, 292, 561-566.

(44) Refaely-Abramson, S., Sharifzadeh, S., Jain, M., Baer, R., Neaton, J. B., Kronik, L. Gap Renormalization of Molecular Crystals from Density-Functional Theory. Phys. Rev. B 2013, 88, 081204.

(45) Sun, H., Ryno, S., Zhong, C., Ravva, M. K., Sun, Z., Körzdörfer, T., Brédas, J. L. Ionization Energies, Electron Affinities, and Polarization Energies of Organic Molecular Crystals: Quantitative Estimations from a Polarizable Continuum Model (PCM)-Tuned Range-Separated Density Functional Approach. J. Chem. Theory Comput. 2016, 12, 2906-2916.

(46) Phillips, H., Zheng, Z. L., Geva, E., Dunietz, B. D. Orbital Gap Predictions for Rational Design of Organic Photovoltaic Materials. Organic Electronics 2014, $15,1509-1520$.

(47) Refaely-Abramson, S., Baer, R., Kronik, L. Fundamental and Excitation Gaps in Molecules of Relevance for Organic Photovoltaics from an Optimally Tuned Range-Separated Hybrid Functional. Phys. Rev. B 2011, 84, 075144. 